Vietnam Journal of Mechanics, Vietnam Academy of Science and Technology, pp. 259-273

DOI:10.15625/0866-7136/9772

\title{
VIBRATIONS OF FRACTIONAL HALF- AND SINGLE-DEGREE OF FREEDOM SYSTEMS
}

\author{
Valentina Ciaschetti ${ }^{1}$, Isaac Elishakoff ${ }^{2, *}$, Alessandro Marzani ${ }^{1}$ \\ ${ }^{1}$ Università degli Studi di Bologna - DICAM, Italy \\ ${ }^{2}$ Florida Atlantic University, Boca Raton, USA \\ *E-mail: elishako@fau.edu \\ Received June 05, 2017
}

\begin{abstract}
In this paper we study vibrations of fractional oscillators by two methods: the triangular strip matrix approach, based on the Grünwald-Letnikov discretization of the fractional term, and the state variable analysis, which is suitable for systems with fractional derivatives of rational order. Some examples are solved in order to compare the two approaches and to conduct comparison with benchmark problems.
\end{abstract}

Keywords: Fractional oscillator, fractional differential equation, numerical solution.

\section{INTRODUCTION}

Oscillators play an important role in scientific and engineering fields, since they represent the simplest model adopted to observe the dynamic behavior of complex structures. In particular, the so called fractional oscillator, that is a generalization of the classical harmonic oscillator in the fractional calculus framework, has started to attract increasing attention in the last decade. It has been found that it can describe many systems by equations consisting of derivatives with fractional order, allowing to obtain more accurate and detailed results. A relevant issue is that the derivative of fractional order at any point of the domain has a local property only when the order is an integer number. For non-integer cases, the fractional derivative is a nonlocal operator and depends on the past values of the function (left derivative) or future ones (right derivative).

Various methods has been developed for the solution of fractional oscillator: Gaul and Schmidt [1] presented a method based on the Grünwald-Letnikov definition of fractional derivatives for numerically evaluating the fractional time-derivatives in conjunction with the time -integration of fractional differential equations is particularly useful for problems which comprise multiple degrees of freedom systems with a high number of time-integration steps and where the immense amount of computational efforts can be drastically reduced without losing the properties and the benefits of fractional

(C) 2017 Vietnam Academy of Science and Technology 
derivatives; Ciesielski and Blaszczyk [2,3] proposed a numerical solution of the nonhomogeneous fractional oscillator equation in a finite time interval that transforms the governing equation into integral form; Yuan and Agrawal [4] converted the fractional differential equation governing the dynamics of the system into a set of differential equations with no fractional derivative terms using Laguerre integral formula, they found that with their method is not necessary to store the past history of the response. Many other techniques take advantage of the fact that the fractional differential equations can be reduced to Volterra type integral equations (see for example Tofighi [5,6], Achar, Hanneken and Clarke $[7,8])$.

As remarked in his paper by Stanislavsky [9], the "free" and "forced" oscillations of a fractional oscillator depend on the index $\alpha$, and they depend on different damping parameters.

In this paper two methods are employed to compare different cases and types of loads. The first one is based on the Grünwald-Letnikov discretization and it involves the so called triangular strip matrices, as it was initially treated by Podlubny [10]; the second one instead refers to the complex eigenanalysis in the state variable domain to decouple the set of FDEs governing the dynamics of multi-degree-of-freedom system, as suggested by Di Paola and Pinnola [11]. In this section, some basic and useful definitions related to fractional calculus are reported.

\section{FRACTIONAL CALCULUS: BACKGROUND AND REPRESENTATION}

Fractional calculus is becoming a very popular subject in the scientific fields. Many books, surveys and researches has been dedicated to this topic. Interested reader can consult for example books by Oldham and Spanier [12], Miller and Ross [13] Gorenflo and Mainardi [14], Samko [15], Kiryakova [16] and Podlubny [17]. This interest stems from the fact that fractional derivatives can be successfully applied to various areas in physics and engineering within the context of the constitutive modeling of materials, control theory, bioengineering or stochastic models. Generally an approximate solution of the problem must be calculated numerically by applying a suitable time-integration scheme; in this case all fractional derivatives or integrals have to be evaluated at any instant of time, so that the entire time history of the respective variables has to be taken into account due to the non-local feature of fractional operators.

There are several alternative representations of the fractional derivatives of order $\alpha$. First of all we mention the Riemann-Liouville representation, involving the equation

$$
{ }_{R L} D_{0}^{-\alpha} f(t)=\frac{1}{\Gamma(m-\alpha)} \frac{d^{m}}{d t^{m}} \int_{0}^{t}(t-\tau)^{m-\alpha-1} f(\tau) d \tau, \quad m-1 \leq \alpha<m \in Z^{+}
$$

where $\Gamma(\cdot)$ is the Gamma function and $Z^{+}$is a set of positive real numbers.

Second representation is the Caputo's fractional derivative definition, that is

$$
{ }_{C} D_{0}^{\alpha} f(t)=\frac{1}{\Gamma(m-\alpha)} \int_{0}^{t}(t-\tau)^{m-\alpha-1} f^{(m)}(\tau) d \tau, \quad m-1 \leq \alpha<m \in Z^{+}
$$


where $f^{(m)}$ is the $m$-th order derivative of $f(t)$.

Another representation of fractional derivatives is based on the Grünwald-Letnikov definition and can be cast in the form.

$$
{ }_{G L} D_{0}^{\alpha} f(t)=\sum_{k=0}^{m-1} \frac{f^{(k)}(0) t^{-\alpha+k}}{\Gamma(-\alpha+k+1)}+\frac{1}{\Gamma(m-\alpha)} \int_{0}^{t}(t-\tau)^{m-\alpha-1} f^{(m)}(\tau) d \tau, \quad m-1 \leq \alpha<m \in Z^{+}
$$

That could be rewritten as

$$
{ }_{G L} D_{0}^{\alpha} f(t)=\lim _{h \rightarrow 0} \frac{1}{h^{\alpha}} \sum_{k=0}^{n}(-1)^{k}\left(\begin{array}{l}
\alpha \\
k
\end{array}\right) f(t-k h) .
$$

It is evident that the only difference between the Riemann-Liouville and the Caputo's derivative definitions is in the sequence of the differentiation. Specifically in the Caputo's case first the function is differentiated $m$ times and then integrated $m-\alpha$ times, while in the Riemann-Liouville case the function is integrated $m-\alpha$ times and then differentiated $m$ times.

\section{GRÜNWALD-LETNIKOV DISCRETIZATION}

A useful method that can be used in order to solve a dynamic vibration problem, i.e. fractional oscillator, by means of fractional calculus, is the Grünwald-Letnikov fractional discretization. This method is based on triangular strip matrix approach to discretize operators of differentiation and integration of arbitrary real order.

The Grünwald-Letnikov operators are important since they can be viewed as the discretized form of Riemann-Liouville or Caputo's fractional operators.

We are starting with the classical backward difference scheme

$$
\left.\left(\frac{d}{d t} f\right)(t)\right|_{t=t_{k}} \cong \frac{1}{h} \nabla f\left(t_{k}\right)=\frac{1}{h}\left[f\left(t_{k}\right)-f\left(t_{k-1}\right)\right], \quad k=1,2, \ldots, N .
$$

All these $N$ equations can be written in matrix form as

$$
\frac{1}{h} \nabla f_{N}=\left[\begin{array}{c}
h^{-1} f(0) \\
h^{-1}\left[f\left(t_{1}\right)-f(0)\right] \\
h^{-1}\left[f\left(t_{k}\right)-f\left(t_{k-1}\right)\right] \\
h^{-1}\left[f\left(t_{N}\right)-f\left(t_{N-1}\right)\right]
\end{array}\right]=\frac{1}{h}\left[\begin{array}{ccccc}
1 & 0 & 0 & \ldots & 0 \\
-1 & 1 & 0 & \ldots & 0 \\
0 & -1 & 1 & \ldots & 0 \\
\vdots & \vdots & \vdots & \ddots & \vdots \\
0 & 0 & 0 & \ldots & 1
\end{array}\right]\left[\begin{array}{c}
f(0) \\
f\left(t_{1}\right) \\
\vdots \\
f\left(t_{k}\right) \\
\vdots \\
f\left(t_{N}\right)
\end{array}\right]
$$

where $f_{N}^{T}=\left[f\left(t_{0}\right), f\left(t_{1}\right), \ldots, f\left(t_{N}\right)\right]^{T}$, and the matrix is a lower triangular strip matrix $B_{N}^{(1)}$ (see Podlubny [10]). According to Podlubny [10], the matrix $B_{N}^{(\alpha)}$ is the discrete analogue of the left-sided fractional differential equation of order $\alpha$.

The second order derivative can be obtained as

$$
\left.\left(\frac{d^{2}}{d t^{2}} f\right)(t)\right|_{t=t_{k}} \cong \frac{1}{h^{2}} \nabla^{2} f\left(t_{k}\right)=\frac{1}{h^{2}}\left[f\left(t_{k}\right)-2 f\left(t_{k-1}\right)+f\left(t_{k-2}\right)\right], \quad k=1,2, \ldots, N
$$


In matrix form Eq. (7) reads

$$
\frac{1}{h^{2}} \nabla^{2} f_{N}=\frac{1}{h^{2}} B_{N}^{(1)} B_{N}^{(1)} f_{N}=B_{N}^{(2)} f_{N},
$$

where $B_{N}^{(2)}$ is a lower triangular matrix again.

Thus, generalizing we can write that

$$
\frac{1}{h^{2}} \nabla^{j} f_{N}=\frac{1}{h^{j}} \underbrace{B_{N}^{(1)} B_{N}^{(1)} \ldots B_{N}^{(1)}}_{j-\text { fold }} f_{N}=B_{N}^{(j)} f_{N} .
$$

The $j$-fold integration proves to be ruled by the inverse operator of $\left(\frac{1}{h} B_{N}^{(j)}\right)^{-1}$, and then

$$
h^{j} I^{j} f_{N}=h^{j} \underbrace{B_{N}^{(1)} B_{N}^{(1)} \ldots B_{N}^{(1)}}_{j-\text { fold }} f_{N} .
$$

The inverse of lower triangular strip matrix is a lower triangular strip matrix too. The latter matrices $B_{N}^{(j)}$ and $\left(B_{N}^{(j)}\right)^{-1}$ may be constructed in the form

$$
\frac{1}{h^{j}} B_{N}^{(j)}=\frac{1}{h^{j}}\left[\begin{array}{cccccccccc}
w_{0} & 0 & \ldots & 0 & 0 & \ldots & \ldots & \ldots & \ldots & 0 \\
w_{1} & w_{0} & \ldots & 0 & 0 & \ldots & \ldots & \ldots & \ldots & 0 \\
\vdots & \vdots & \ddots & \vdots & \ddots & \ddots & \ddots & \ddots & \ddots & \vdots \\
w_{j} & w_{j-1} & \ddots & w_{0} & \vdots & \vdots & \ddots & \ddots & \ddots & \vdots \\
0 & w_{j} & \ddots & \vdots & w_{0} & \vdots & \ddots & \ddots & \ddots & \vdots \\
\vdots & \vdots & \ddots & w_{j-1} & \vdots & w_{0} & \ddots & \ddots & \ddots & \vdots \\
\vdots & \vdots & \ddots & \ddots & w_{j-1} & \vdots & \ddots & \ddots & \ddots & \vdots \\
\vdots & \vdots & \ldots & \ddots & \ddots & \ddots & \ddots & \ddots & \ddots & \vdots \\
\vdots & \vdots & \ldots & \ddots & \ddots & \ddots & w_{j-1} & \ddots & \ddots & 0 \\
0 & 0 & \ldots & 0 & 0 & \ldots & w_{j} & w_{j-1} & \ldots & w_{0}
\end{array}\right],
$$

with $\omega_{j}=(-1)^{r}\left(\begin{array}{c}j \\ r\end{array}\right), r=0,1, \ldots j, \gamma_{0}=1, \gamma_{r}=r$.

\section{UNIT-STEP RESPONSE OF HALF-DEGREE-OF-FREEDOM SYSTEMS}

Let us try to apply this methodology on a simplest possible problem of the half degree of freedom oscillator, i.e. oscillator with damping and spring but no mass, unitstep response by means of Wolfram Mathematica 9 package. We are dealing with following problem:

$$
c_{\alpha}\left(D^{\alpha} x\right)(t)+k x(t)=U(t),
$$

in which $c_{\alpha}=\frac{1}{2}$ is the damping coefficient, $k=1$ is the elastic coefficient, $U(t)=$ unitstep force and $0<\alpha<1$. 
Using the Grunwald-Letnikov approach, the response history $x(t)$ at each discrete nodal point $x(j \Delta)$, depicting the behavior of the oscillator during time, can be found.

In particular, the solution of the above problem reads

$$
\frac{1}{\Delta t} c_{\alpha}+B_{N}^{(\alpha)} x_{N}+k_{k} x_{N}=f_{N}
$$

with $\Delta t=h$ is the temporal step, $N \Delta t$ is the total considered time. Also, vectors

$$
\begin{aligned}
& x_{N}=[x(\Delta t), x(2 \Delta t), \ldots, x(N \Delta t)]^{T}, \\
& f_{N}=[f(\Delta t), f(2 \Delta t), \ldots, f(N \Delta t)]^{T},
\end{aligned}
$$

and $B_{N}^{(\alpha)}$ are defined as before.

Thus the response is

$$
x_{N}=\left[\frac{c_{\alpha}}{\Delta t} B_{N}^{(\alpha)}+k_{k} I\right]^{-1} f_{N}
$$

with $0<\alpha<1$.

In particular, if we define the mesh points as

$$
t_{j}=a+j \Delta t, \quad j=0,1, \ldots, N .
$$

The discrete approximation of Grünwald-Letnikov is

$$
D_{G-L}^{\alpha, \Delta t}=\frac{1}{\Delta t^{\alpha}} \sum_{k=0}^{j} \omega_{k}^{(\alpha)} x\left(t_{j-k}\right),
$$

where

$$
\omega_{k}^{(\alpha)}=(-1)^{k}\left(\begin{array}{l}
\alpha \\
k
\end{array}\right)=(-1)^{k} \frac{\alpha(\alpha-1) \ldots(\alpha-k+1)}{k !}=\frac{\Gamma(k-\alpha)}{\Gamma(-\alpha) \Gamma(k+1)} .
$$

\section{APPLICATIONS}

In the following some numerical examples are reported in order to understand how the behavior of the half degree of freedom is affected by the change of the fractional order value $\alpha$.

In the numerical implementation, we assume a time step $\Delta t=0.02$, and $c_{\alpha}=$ $1 / 2, k=1$ and $\alpha$ taken as either of following values: $1 / 2,1 / 3,2 / 3,1$.

For different values of $\alpha$ we can observe that the higher the order of differentiation the higher the response curve (Fig. 1).

We can also compare the response of the half-oscillator for different types of loading (Fig. 2). 


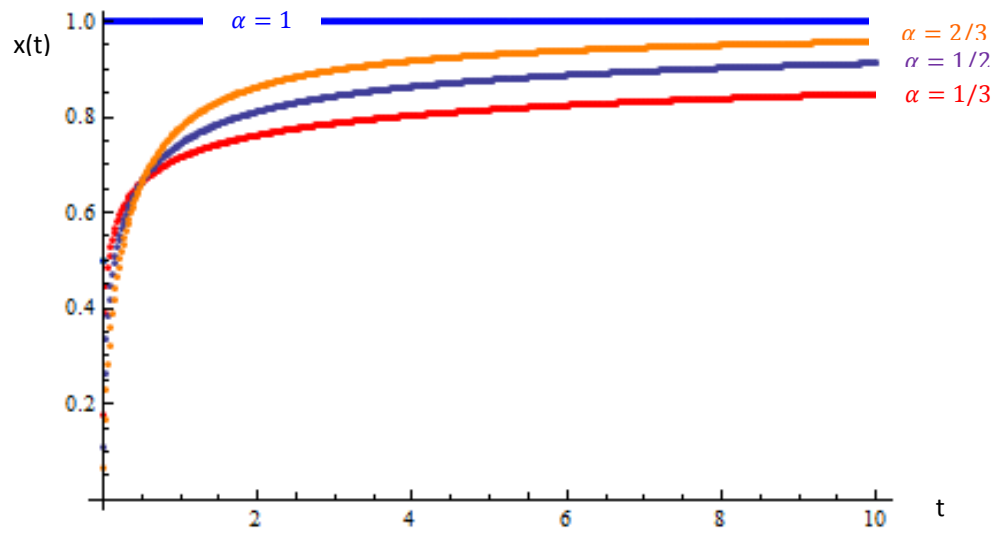

Fig. 1. Half-degree of freedom fractional oscillator comparing different fractional orders of differentiation

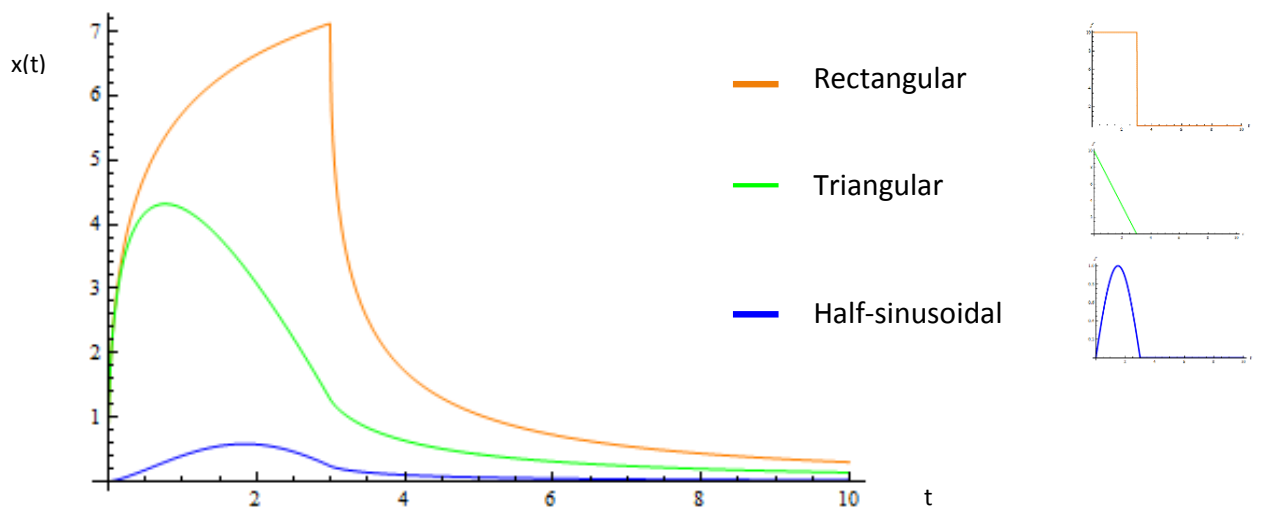

Fig. 2. Comparison of responses to different types of loading: rectangular, triangular, half-sinusoidal excitations

\section{UNIT-STEP RESPONSE OF A SINGLE DOF FRACTIONAL OSCILLATOR}

Let us consider now the unit-step response of a fractionally damped oscillator. The general equation of motion can be written in the form

$$
m \ddot{x}(t)+c_{\alpha}\left(D^{\alpha} x\right)(t)+k x(t)=U(t) .
$$

This equation describes the dynamics of a single-degree-of-freedom spring-massdamper system, that represents the model of frequency dependent viscoelastic damping of a material. In this case the Grünwald-Letnikov solution can be found in the following recurrent form

$$
M \Delta t^{2} B_{N}^{(2)} x_{N}+C_{\alpha} \Delta t^{\alpha} B_{N}^{(\alpha)} x_{N}+K x_{N}=f_{N},
$$

where $B_{N}^{(2)}=B_{N}^{(1)^{2}}$, and $B_{N}^{(1)}$ is defined as before. 
Then the vector of solution $x_{N}$ is given as

$$
x_{N}=\left(M \Delta t^{2} B_{N}^{(2)}+C_{\alpha} \Delta t^{\alpha} B_{N}^{(\alpha)}\right)^{-1} f_{N}
$$

in which the matrix in parenthesis is a lower triangular strip matrix.

Some example was implemented in Mathematica, by changing the input values (Figs. 3-4)

$x(t)$

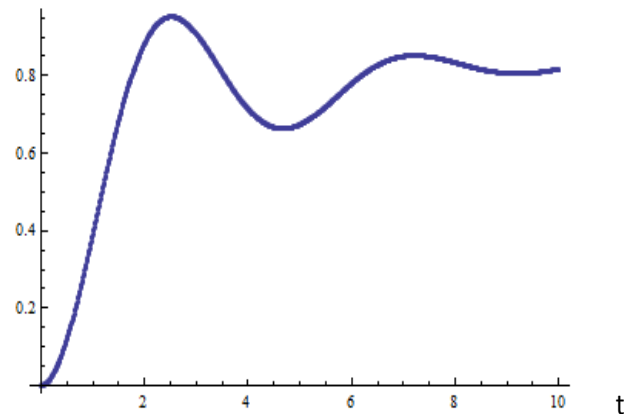

(a)

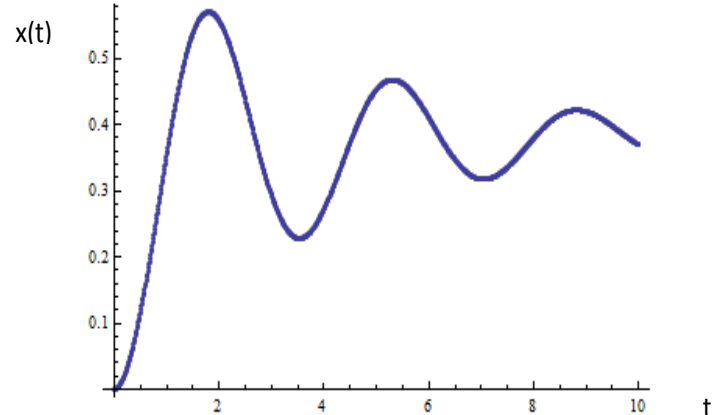

(b)

Fig. 3. Single-degree-of-freedom system simulation for parameters set at (a) $m=1, c=1, k=1, \alpha=1 / 2$; (b) $m=1, c_{\alpha}=\pi / 4, k_{k}=\pi^{2} / 4, \alpha=1 / 2$

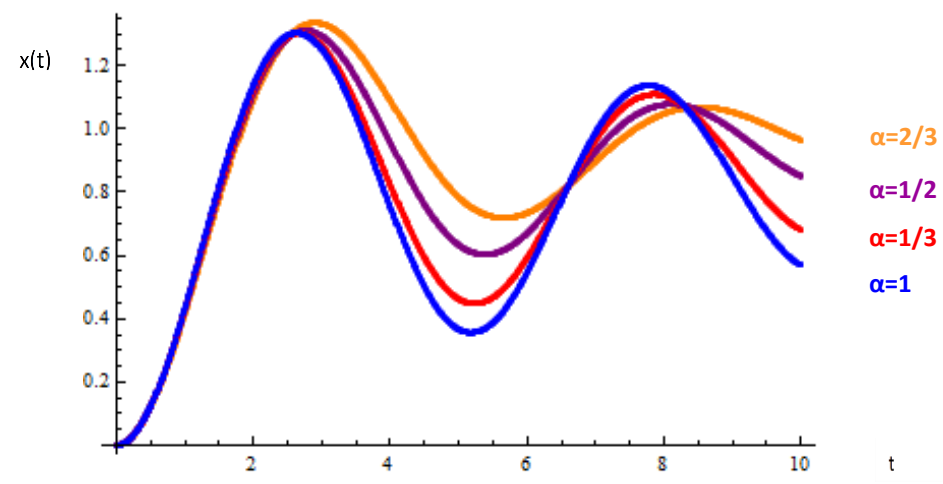

Fig. 4. Single-degree-of-freedom system simulation with parameters set at $m=1, c_{\alpha}=1, k=1, \alpha=1 / 2,1 / 3,2 / 3,1$

\section{STATE VARIABLE ANALYSIS}

Another novel method that can be used to solve this problem is based on complex eigenanalysis in the state variable domain, to uncouple the set of rational order fractional differential equations governing the dynamics of the system. 
The limitation of this method consists of the following fact: since fractional orders are involved, the state variable analysis can be performed for fractional degree-offreedom systems (FSDOF) with the derivative order being rational.

The fractional $r$-degree-of-freedom system is described by a coupled system of fractional differential equations of various FSDOF; the problem can be cast in the form

$$
M \ddot{x}(t)+\sum_{i=1}^{l} C_{i} D^{\beta_{i}} x(t)+K x(t)=f(t)
$$

where $M$ and $K$ are the mass and the stiffness matrices respectively, $C_{i}$ is the matrix of the coefficients $c_{\beta_{i}}$ of the involved fractional terms of order $\beta_{i}, x(t)$ is the response vector and $f(t)$ is the vector of loading.

We assume that all fractional orders are rational, it is possible to represent the generic fractional order of the above equation as $\beta_{i}=a_{i} / b_{i}$ where $a_{i}, b_{i} \in \mathbb{N}$ with $i=1,2 \ldots, l$. Thus the above system can be rewritten as a sequential liner differential equations of fractional orders

$$
\sum_{j=1}^{n} C_{j} D^{j \alpha} x(t)+K x(t)=f(t),
$$

where $\alpha$ is chosen such that $n \alpha$ equals the maximum order that appears in the system of equations and such that all involved orders can be represented as as $\beta_{i}=d_{i} \alpha$, where $d_{i} \in \mathbb{N}$. In this case of $n \alpha=2$ and the corresponding matrix $C_{n}=M$ is the matrix of the mass; all matrices in the latter equation have dimension $r \times r$. Introducing then the vector of state variables

$$
z^{T}(t)=\left[x^{T}(t) D^{\alpha} x^{T}(t) D^{2 \alpha} x^{T}(t) \ldots D^{\alpha(n-1)} x^{T}(t)\right]
$$

and considering the $n-1$ fractional terms in Eq. (22) we finally get an identity

$$
\begin{aligned}
\sum_{j=1}^{n-1} C_{j+1} D^{\alpha} D^{(j-1) \alpha} x(t) & =\sum_{j=1}^{n-1} C_{j+1} D^{j \alpha} x(t), \\
\sum_{j=1}^{n-2} C_{j+2} D^{\alpha} D^{(j-1) \alpha} x(t) & =\sum_{j=1}^{n-2} C_{j+2} D^{j \alpha} x(t), \\
& \vdots \\
C_{n} D^{\alpha} x(t) & =C_{n} D^{\alpha} x(t) .
\end{aligned}
$$

Then the set of $r \times n$ coupled differential equations is readily cast in the form

$$
A D^{\alpha} z(t)+B z(t)=g(t),
$$


where $g^{T}(t)=\left[\begin{array}{llll}f^{T}(t) & 0 & \ldots & 0\end{array}\right], \quad A$ and $B$ are symmetric matrices defined as

$$
\begin{gathered}
A=\left[\begin{array}{ccccc}
C_{1} & C_{2} & \ldots & C_{n-1} & C_{n} \\
C_{2} & C_{3} & & C_{n} & 0 \\
\vdots & & \ddots & \vdots & \\
C_{n-1} & C_{n} & \ldots & 0 & 0 \\
C_{n} & 0 & & 0 & 0
\end{array}\right], \\
B=\left[\begin{array}{ccccc}
K & 0 & \ldots & 0 & 0 \\
0 & -C_{2} & & -C_{n-1} & -C_{n} \\
& \vdots & \ddots & \vdots & \\
0 & -C_{n-1} & \ldots & 0 & 0 \\
0 & -C_{n} & & 0 & 0
\end{array}\right] .
\end{gathered}
$$

Decomposing $z(t)$ in the orthogonal basis of eigenvectors of A and B and making the complex modal transformation

$$
\begin{gathered}
\Psi^{T} \mathrm{~A} \Psi=U_{D}, \quad \Psi^{T} \mathrm{~B} \Psi=V_{D}, \\
z(t)=\Psi y(t),
\end{gathered}
$$

with $U_{D}, V_{D}$ being diagonal matrices, a new set of decoupled fractional differential equation is derived in the form

$$
U_{D} D^{\alpha} y(t)+V_{D}, \quad y(t)=\mu(t)
$$

where $\mu(t)=\Psi^{T} g(t)$. solved.

Once the decoupled set is found, the fractional differential equations can be readily

Even if the state of variable problem in Eq. (29) has a greater number of involved variables with respect to the problem cast in terms of displacements in Eq. (25) of the nodal analysis, this apparent increase of computational burden is balanced by the fact that the maximum involved order in the state variable domain is smaller than the maximum order in the nodal analysis. Moreover, in Eq. (22) the system is a set of coupled in the general case, while the system in Eq. (25) leads readily to the set of uncoupled equations in Eq. (29).

In order to make a comparison between the two methods, let us reconsider the same problems of before.

The results implemented in Matlab are in perfect agreement with the ones obtained with the triangular strip matrix approach derived through use of Mathematica software (Fig. 5)

It is important to notice that, when the order $\alpha=1$ the state variables vector is the classical one: $z^{T}(t)=\left[\begin{array}{ll}x^{T}(t) & \dot{x}\end{array}\right]$.

Although the method is applicable exactly if the involved orders are rational, even in the general case of $\alpha \in \mathbb{R}$. It could lead to reasonable results by approximating $\alpha$ as a fraction. 

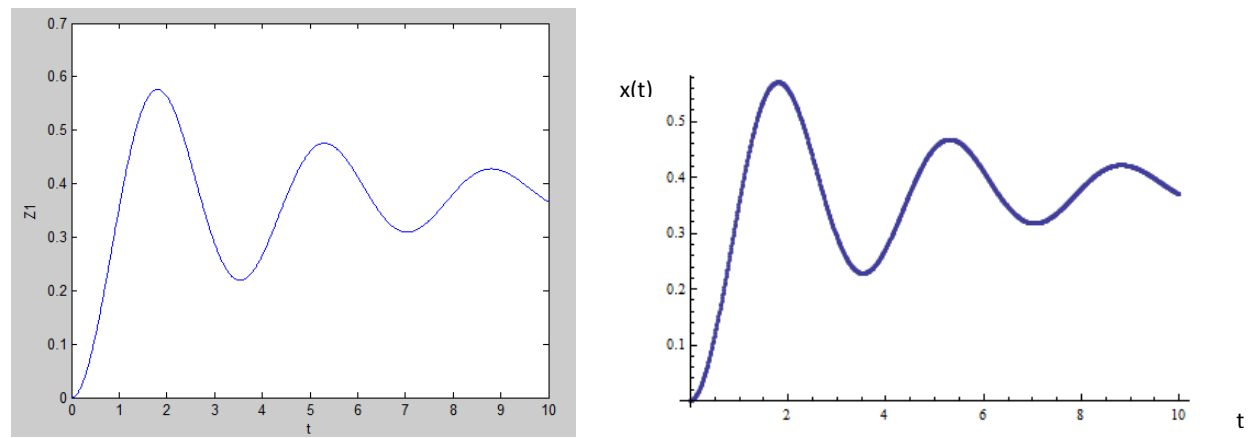

Fig. 5. Unit-step response of SDOF system with $\alpha=1 / 2$ in MATLAB (left) and Mathematica (right)

\section{RESPONSE TO IMPULSIVE LOADS}

Impulsive forces are forces of large magnitude that acts over a short period of time. With the state variable analysis, in order to get the response for any non-unitary driving force, a discrete convolution is necessary. If the Duhamel integration method is applied, the solution obtained before with the Mittag-Leffler function [10,17,18], since correspond to the unitary step, should be derivated and then applied as kernel in the code.

In particular, using the Green function

$$
G(t)=\frac{1}{m} \sum_{j=0}^{\infty}-\frac{1}{j !}\left(\frac{k}{m}\right)^{j} t^{2 j+1} E_{2-\beta, 2+j \beta}^{(j)}\left(-\frac{c_{\beta}}{m} t^{2-\beta}\right),
$$

to solve the governing equation, where

$$
E_{\gamma, \mu}^{(j)}(z)=\frac{d^{j}}{d z^{j}} E_{\gamma, \mu}(z)=\sum_{l=0}^{\infty} \frac{(l+j) ! z^{l}}{l ! \Gamma(\gamma l+\gamma j+\mu)},
$$

is the derivative of order $\mathrm{j}$ of the two parameters Mittag-Leffler function, assuming the system is quiescent at $t=0$, the solution is given as

$$
x(t)=\int_{0}^{t} G(t-\tau) f(\tau) d \tau .
$$

This solution can be computationally demanding since there are two summations with infinity terms as kernel in the convolution integral.

In the following we report the results for rectangular, triangular and half sinusoidal impulsive loadings, obtained with both methods (Figs. 6-8).

\section{Case 1: Rectangular load}

In the particular case of rectangular load depicted in Fig. 6, the response id portrayed in Fig. 7. The results are obtained by MATLAB code as well as symbolic manipulation package MATHEMATICA. 


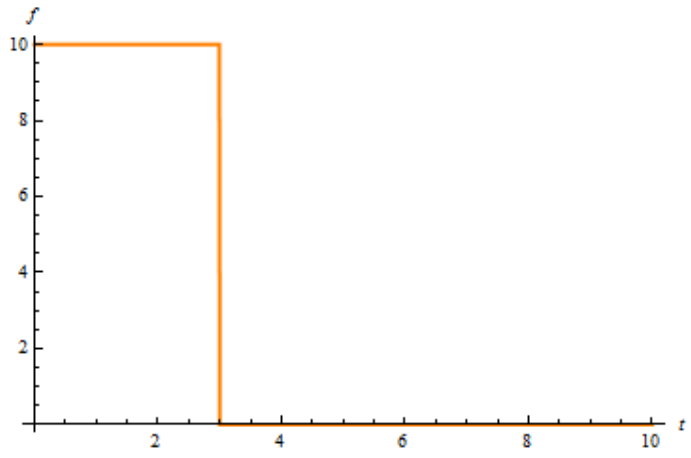

DATA

$m=1$;

$c \alpha=\pi / 4 ;$

$k=\pi^{2} / 4 ;$

$\beta=1 / 2$

$p=10$;

Fig. 6. Timewise rectangular load
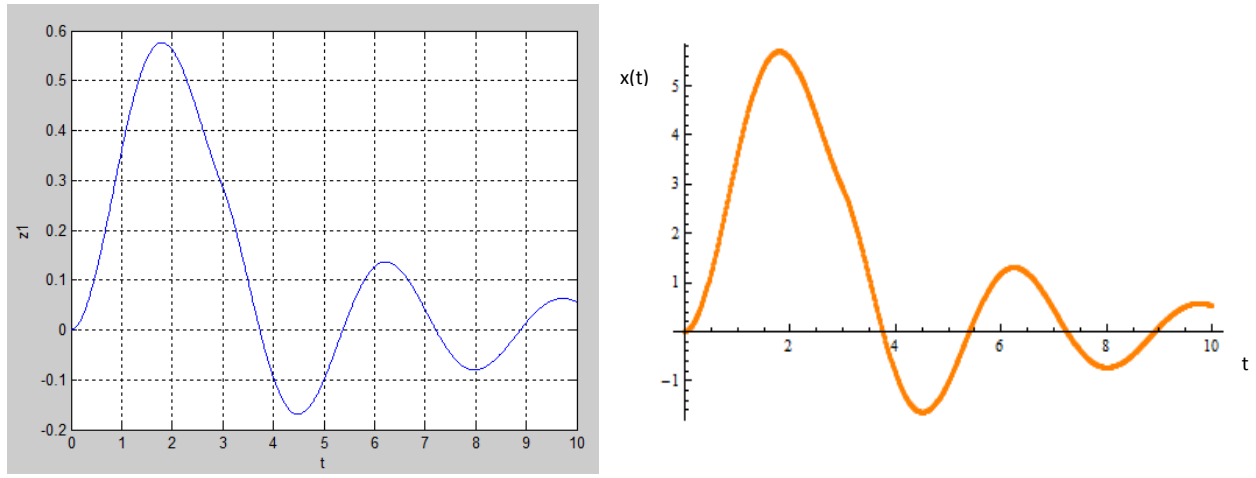

Fig. 7. Response of SDOF system to rectangular load with MATLAB (left) and Mathematica (right)

\section{Case 2: Triangular load}

In the case of triangular load in time (Fig. 8), we obtain responses shown in Fig. 9.

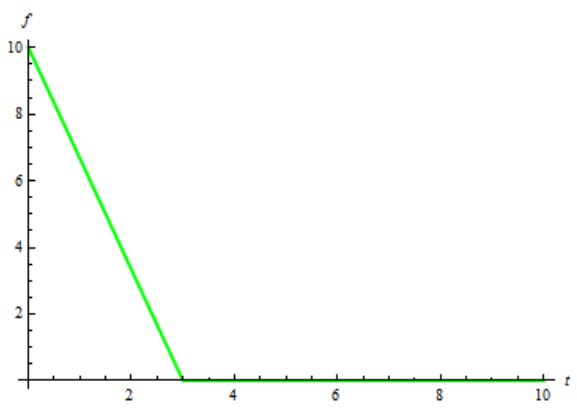

DATA
$\mathrm{m}=1 ;$
$\mathrm{c} \alpha=\pi / 4 ;$
$\mathrm{k}=\pi^{2} / 4 ;$
$\beta=1 / 2 ;$
$\mathrm{p}=10$

Fig. 8. Timewise triangular load 

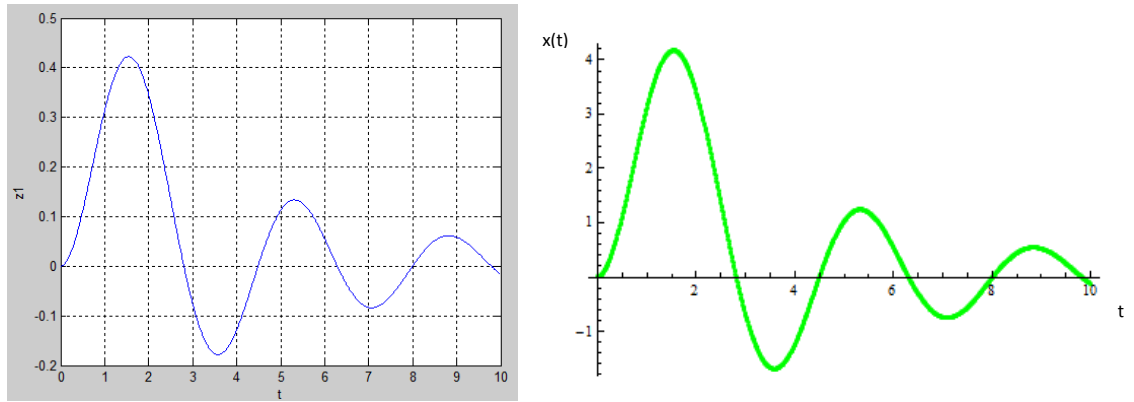

Fig. 9. Response of SDOF system to triangular load with MATLAB (left) and Mathematica (right)

\section{Case 3: Half-sinusoidal load}

In the particular case of half-sinusoidal load, shown in Fig. 10 the obtained results are depicted in Fig. 11.

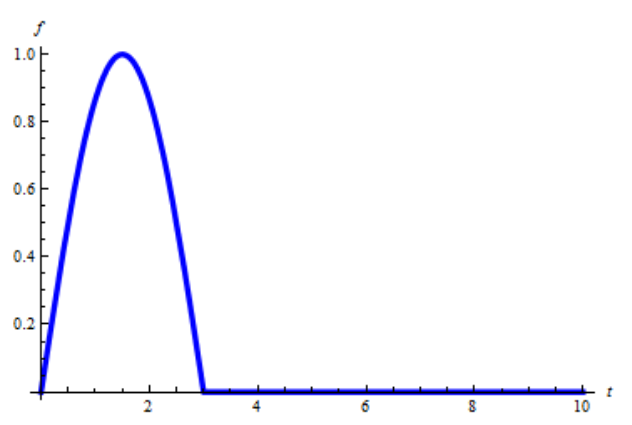

$$
\begin{aligned}
& \text { DATA } \\
& \mathrm{m}=1 ; \\
& \mathrm{c} \alpha=\pi / 4 ; \\
& \mathrm{k}=\pi^{2} / 4 ; \\
& \beta=1 / 2 ; \\
& \mathrm{p}=10 ;
\end{aligned}
$$

Fig. 10. Timewise half-sinusoidal load
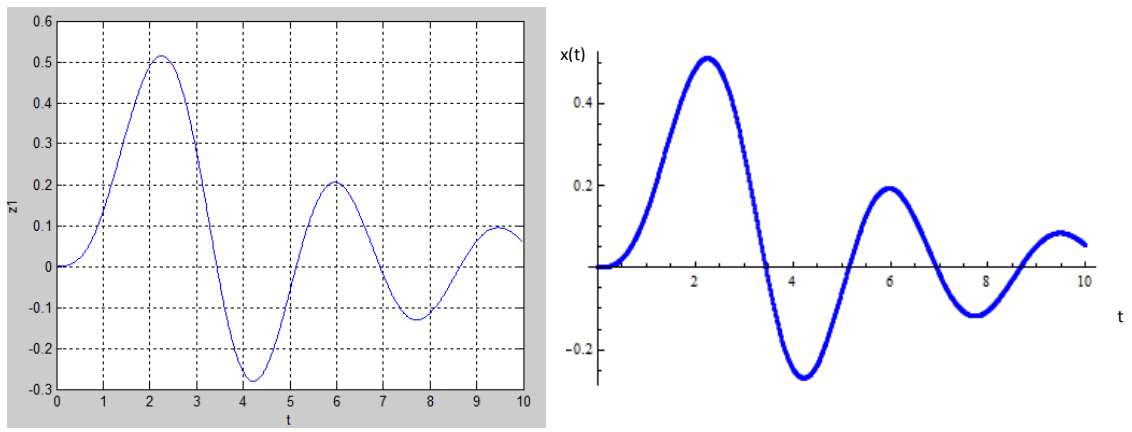

Fig. 11. Response of SDOF system to half-sinusoidal load with MATLAB (left) and Mathematica (right) 

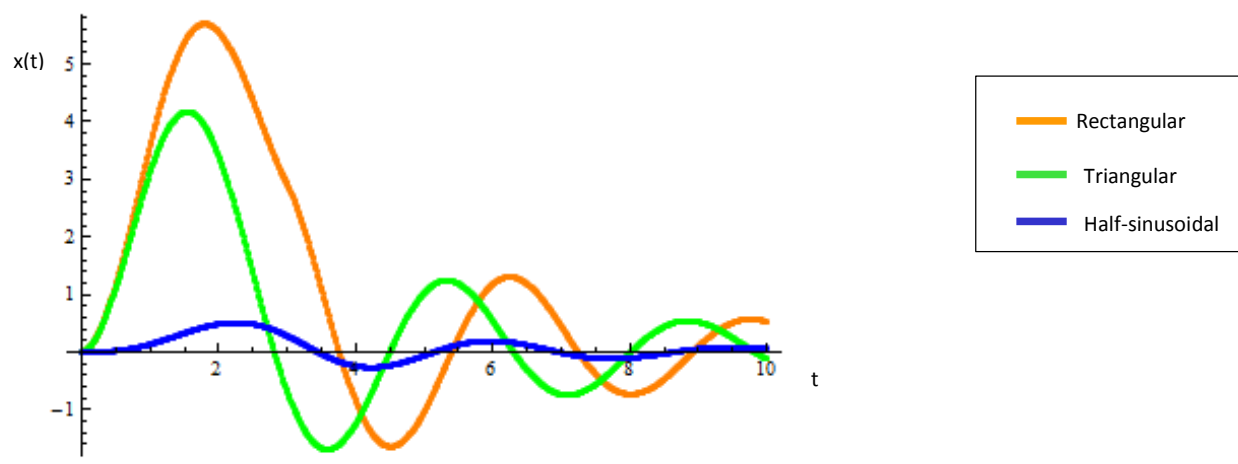

Fig. 12. Responses of SDOF system to different loading types obtained by Mathematica code

\section{CLOSED-FORM SOLUTION OF THE RESPONSE}

Let us consider the general equation of motion for a fractional single-degree-offreedom system

$$
\ddot{x}(t)+C_{\alpha}\left(D^{\alpha} x\right)(t)+\omega_{0}^{2} x(t)=f(t),
$$

where $C_{\alpha}=c_{\alpha} / m, \omega_{0}^{2}=k / m, f(t)=F(t) / m$.

The solution of Eq. (33) is obtained following the method of Miller and Ross [13], and used also by Agrawal [19] and Freundlich and Tylikowski [20]. We assume the forcing function as

$$
F(t)=A \cdot e^{\omega t},
$$

where $A$ is the amplitude and $\omega$ the frequency, and zero initial conditions. The Eq. (32) represents the solution of the differential equation with the Green's function, when $\alpha=$ 0.5 in the form of

$$
G(t)=\sum_{k=1}^{4} \frac{\lambda_{k}}{4 \lambda_{k}^{3}+C_{\alpha}} e^{\lambda_{k}^{2} t} \cdot\left[1+\operatorname{erf}\left(\lambda_{k} \sqrt{t}\right)\right],
$$

in which $\lambda_{k}$ are the distinct complex roots of the characteristic polynomial

$$
P(z)=z^{4}+C_{\alpha} z+\omega_{0}^{2}=0,
$$

and erf $\left(\lambda_{k} \sqrt{t}\right)$ is the error function defined as

$$
\operatorname{erf}\left(\lambda_{k} \sqrt{t}\right)=\frac{2}{\sqrt{\pi}} \int_{0}^{x} e^{-t^{2}} d t
$$

Substituting the expression of the Green function in the Eq. (32) leads

$$
\left.x(t)=\frac{A}{m} \int_{0}^{t} \sum_{k=1}^{4} \frac{\lambda_{k}}{4 \lambda_{k}^{3}+C_{\alpha}} e^{\lambda_{k}^{2}(t-\tau)} \cdot\left[1+\operatorname{erf}\left(\lambda_{k} \sqrt{(t-\tau}\right)\right)\right] e^{\omega(\tau)} d \tau .
$$


In the case of classical first order derivative damping model, the system response is

$$
x(t)=\frac{A}{m \omega} \int_{0}^{t} e^{-h(t-\tau)} \sin (\omega(t-\tau)) e^{\omega(\tau)} d \tau,
$$

with $\omega=\sqrt{\omega_{0}^{2}+h^{2}}$.

The solution of Eq. (38) becomes

$$
x(t)=\frac{A e^{-h t+\omega \tau}\left(e^{h t} \omega-\omega \cos (t \omega)-h \sin (t \omega)\right)}{m \omega\left(h^{2}+\omega^{2}\right)} .
$$

\section{CONCLUSION}

This paper deals with determination of vibrational behavior of half- and singledegree of freedom via fractional calculus. It appears interesting to extend the analysis to multi-degree of freedom systems in light of their possible probabilistic analysis framework.

\section{ACKNOWLEDGEMENTS}

This work was performed at the Florida Atlantic University, as a part of Master's thesis of V.C. under the direction of I.E and A.M. We are thankful to Dr. Francesco Pinnola of the University of Palermo, Italy, for providing us different materials and references.

\section{REFERENCES}

[1] A. Schmidt and L. Gaul. On the numerical evaluation of fractional derivatives in multi-degree-of-freedom systems. Signal Processing, 86, (10), (2006), pp. 2592-2601. doi:10.1016/j.sigpro.2006.02.006.

[2] M. Ciesielski and T. Błaszczyk. Numerical solution of non-homogenous fractional oscillator equation in integral form. Journal of Theoretical and Applied Mechanics, 53, (2015), pp. 959-968. doi:10.15632/jtam-pl.53.4.959.

[3] T. Blaszczyk and M. Ciesielski. Fractional oscillator equation-transformation into integral equation and numerical solution. Applied Mathematics and Computation, 257, (2015), pp. 428435. doi:10.1016/j.amc.2014.12.122.

[4] L. Yuan and O. P. Agrawal. A numerical scheme for dynamic systems containing fractional derivatives. Journal of Vibration and Acoustics, 124, (2), (2002), pp. 321-324. doi:10.1115/1.1448322.

[5] A. Tofighi. An especial fractional oscillator. International Journal of Statistical Mechanics, 2013, (2013). doi:10.1155/2013/175273.

[6] A. Tofighi. The intrinsic damping of the fractional oscillator. Physica A: Statistical Mechanics and its Applications, 329, (1), (2003), pp. 29-34. doi:10.1016/s0378-4371(03)00598-3.

[7] B. N. N. Achar, J. W. Hanneken, and T. Clarke. Damping characteristics of a fractional oscillator. Physica A: Statistical Mechanics and its Applications, 339, (3), (2004), pp. 311-319. doi:10.1016/j.physa.2004.03.030.

[8] B. N. N. Achar, J. W. Hanneken, and T. Clarke. Response characteristics of a fractional oscillator. Physica A: Statistical Mechanics and its Applications, 309, (3), (2002), pp. 275-288. doi:10.1016/s0378-4371(02)00609-x. 
[9] A. A. Stanislavsky. Fractional oscillator. Physical Review E, 70, (5), (2004). doi:10.1103/physreve.70.051103.

[10] I. Podlubny. Matrix approach to discrete fractional calculus. Fractional Calculus and Applied Analysis, 3, (4), (2000), pp. 359-386.

[11] M. Di Paola, F. P. Pinnola, and P. D. Spanos. Analysis of multi-degree-of-freedom systems with fractional derivative elements of rational order. In International Conference on Fractional Differentiation and Its Applications (ICFDA). IEEE, (2014), pp. 1-6. doi:10.1109/icfda.2014.6967364.

[12] K. B. Oldham and J. Spanier. The fractional calculus. Academic Press, New York, (1974).

[13] K. S. Miller and B. Ross. An introduction to the fractional calculus and fractional differential equations. John Wiley and Sons, Inc., New York, (1993).

[14] R. Gorenflo and F. Mainardi. Fractional calculus: Integral and differential equations of fractional order, pp. 223-276. Springer Verlag, (1997).

[15] S. G. Samko, A. A. Kilbas, and O. I. Marichev. Fractional integrals and derivatives-theory and applications. Gordon and Breach Science Publishers, Longhorne, PA, USA, (1993).

[16] V. S. Kiryakova. Generalized fractional calculus and applications. Longman Scientific \& Technical, Longman House, Burnt Mill, Harlow, England, (1993).

[17] I. Podlubny. Fractional differential equations. Academic Press, New York, (1999).

[18] A. M. Mathai and H. J. Haubold. Mittag-Leffler functions and fractional calculus, chapter 2, pp. 79-134. Springer, (2008). doi:10.1007/978-0-387-75894-7_2.

[19] O. P. Agrawal. Analytical solution for stochastic response of a fractionally damped beam. Journal of Vibration and Acoustics, 126, (4), (2004), pp. 561-566. doi:10.1115/1.1805003.

[20] J. Freundlich and A. Tylikowski. Transient resonance oscillations of a system with fractional derivative damping of order 1/2. Machine Dynamics Research, 37, (4), (2015), pp. 27-33. 